\title{
Temperature Dependence of the Electron and Hole Scattering Mechanisms in Silicon Analyzed through a Full-Band, Spherical-Harmonics Solution of the BTE
}

\author{
SUSANNA REGGIANI, MARIA CRISTINA VECCHI and MASSIMO RUDAN* \\ Dipartimento di Elettronica, Informatica e Sistemistica, Università di Bologna, Viale Risorgimento 2, 40136 Bologna, Italy
}

\begin{abstract}
By adopting the solution method for the BTE based on the spherical-harmonics expansion (SHE) [1], and using the full-band structure for both the electron and valence band of silicon [2], the temperature dependence of a number of scattering mechanisms has been modeled and implemented into the code HARM performing the SHE solution. Comparisons with the experimental mobility data show agreement over a wide range of temperatures. The analysis points out a number of factors from which the difficulties encountered in earlier investigations seemingly originate, particularly in the case of hole mobility.
\end{abstract}

Keywords: Carrier mobility, scattering mechanisms, Boltzmann equation, spherical-harmonics expansion

\section{INTRODUCTION}

We present here an investigation of the temperature dependence of the scattering mechanisms described at microscopic level through the solution of the BTE based on an expansion in spherical harmonics. Such investigation is also relevant from the application viewpoint because it allows for a detailed analysis of the temperature and impurity dependence of mobility, hence of the performance of the submicron and power semiconductor devices. The mechanisms considered here are acoustical- and optical-phonon scattering, ionized-impurity scattering, and impact ionization. In particular, phonon and impurity scattering have a strong influence on mobility also at low energies. In addition, the effect of incomplete ionization of dopant has been introduced as well, to account for the behavior of carrier mobility at high impurity concentration and low temperatures.

By this approach the experimental mobility curves of electrons and holes are well reproduced in a wider range of temperatures and impurity concentrations than in earlier investigations.

\footnotetext{
*Corresponding author. Tel.: +39(51) 64430-16, Fax:64430-73. e-mail: mrudan@deis.unibo.it.
} 


\section{PHYSICAL MODELS}

The mathematical model resulting from the SHE of the BTE is briefly summarized here, in the homogeneous case, with reference to both the conduction and valence band:

$$
\begin{aligned}
-q^{2} F^{2} \frac{\partial}{\partial E}[ & \\
& \left.g(E) u_{g}^{2}(E) \frac{\partial f_{0}}{\partial E}\right]= \\
& +3 c_{\mathrm{op}} g(E) \mathcal{M}_{\mathrm{op}}-3 c_{\mathrm{ii}} g^{2}(E) f_{0}(E) \\
& +3 g(E) \int A\left(E^{\prime}, E\right) f_{0}\left(E^{\prime}\right) g\left(E^{\prime}\right) d E^{\prime}
\end{aligned}
$$

where $\mathcal{M}_{\mathrm{op}}=\left(N_{\mathrm{op}}^{+} f_{0}^{+}-N_{\mathrm{op}} f_{0}\right) g^{+}-\left(N_{\mathrm{op}}^{+} f_{0}-N_{\mathrm{op}}\right.$ $\left.f_{0}^{-}\right) g^{-}$. The symbols have the following meaning: $g(E)$ is the density of states, $u_{\mathrm{g}}(E)$ the modulus of the group velocity, $\tau$ the total scattering rate, $F$ the electric field, $c_{\text {op }}$ a constant proportional to the optical-phonon coupling constant, $N_{\text {op }}$ the opticalphonon occupation number, $N_{\mathrm{op}}^{+}=N_{\mathrm{op}}+1$, $g^{ \pm}(E)=g\left(E \pm \hbar \omega_{\text {op }}\right)$, where $\hbar \omega_{\text {op }}$ is the opticalphonon energy, and similarly for $f_{0}^{ \pm}(E)$. Impact ionization is also considered: $c_{\mathrm{ii}} g(E)$ is the total impact-ionization scattering rate and $A\left(E^{\prime}, E\right)$ is a suitable kernel [3]. All the scattering mechanisms considered here exhibit a temperature dependence:

- acoustical-phonon scattering shows an explicit dependence on temperature [4];

- optical-phonon scattering depend on temperature through the occupation number $N_{\text {op }}$ [4];

- ionized-impurity scattering depend on temperature through the partial ionization effect;

- impact ionization depend on temperature through the energy gap.

In the following we will focus on the effect of temperature on the carrier mobility as a function of impurity concentration, therefore we will describe with more details the scattering term strongly related to this kind of collision.

The ionized-impurity scattering is described according to the Brooks-Herring (BH) model:

$$
\begin{aligned}
S_{\mathrm{BH}}= & \frac{N_{i} e^{2} I^{2}}{(2 \pi)^{2} \hbar}\left[\frac{Z e}{\varepsilon_{\mathrm{s}}\left(2 k^{2}(1-\cos \xi)+\beta^{2}\right)}\right]^{2} \\
& \times \delta\left(E^{\prime}-E\right)
\end{aligned}
$$

where $N_{\mathrm{i}}$ is the ionized-impurity concentration, $I$ is the overlap factor, $Z e$ the impurity charge, $\varepsilon_{\mathrm{s}}$ the semiconductor dielectric constant, $k$ the wave vector magnitude, $\xi$ the scattering angle, $\beta$ the inverse screening length.

The overlap factor accounts for the interaction between the initial and final states in a collision event and depends on the shape of the band. According to [4], it is considered constant for the conduction band, incorporating its value in the coupling constant of each of the scattering mechanisms. For the valence band the expression obtained by Wiley [4] has been adopted:

$$
I^{2}\left(k, k^{\prime}\right)=\frac{1}{4}\left(1+3 \cos ^{2} \xi\right)
$$

which produces an additional angular dependence in the spherical harmonics expansion of the impurity scattering term, while, for the collisions with phonons the expansion of the scattering terms turns out to be unmodified, apart from a constant factor.

The inverse screening length is usually given by the Debye expression which is obtained by solving Poisson's equation considering an isotropic parabolic band and Boltzmann statistics. In this work we consider a more complicated description of the band structure, based on pseudo-potential calculation, and therefore we compute the inverse screening length following the general definition [5]:

$$
\beta^{2}=\frac{e^{2}}{\varepsilon_{\mathrm{s}} K_{\mathrm{B}} T_{0}} \int g(E) f_{0}(E)\left(1-f_{0}(E)\right) d E .
$$

where $e$ electron charge, $K_{\mathrm{B}}$ Boltzmann constant, $T_{0}$ lattice temperature, $g(E)$ density of states, $f_{0}(E)$ first term of SHE of distribution function. It is necessary to include additional corrections to the Brooks-Herring (2) scattering term to take into account the effects of the Born approximation of order higher than 1 for incoherent collision with a 
single impurity and for coherent collisions with impurity-pairs. The theory of multiple collisions of [6], in agreement with the Brooks-Herring formulation, considers an electron gas interacting with $N_{\mathrm{i}}$ impurities in the crystal. The main difference between the results of [6] and that of Brooks-Herring is that the latter considers the potential of a single impurity, resulting in a total collision probability equal to $N_{\mathrm{i}}$ times the collision probability of a single impurity. The result of [6], instead, keeps the information on the interaction potential between electrons and impurities, which is expressed by $\sum_{j=1}^{N} V\left(\mathbf{r}-\mathbf{R}_{j}\right)$, with $\mathbf{R}_{j}$ position vector for the $j$-th ion, and modifies the scattering matrix in such a way that it becomes:

$$
S\left(E, E^{\prime}\right)=S_{\mathrm{BH}}\left(E, E^{\prime}\right)\left[1+\delta_{\mathrm{M}}+\delta_{\mathrm{D}}\right]
$$

where $\delta_{\mathrm{M}}, \delta_{\mathrm{D}}$ are multiple collisions and impurity dressing constants.

The effect of impurity clustering, relevant for high doping concentrations, has also been taken into account. At high doping densities it is described as if the carrier scatters with a cluster of $Z$ ions, with $Z$ number of charge. The partial ionization, relevant for both high doping concentration and low temperature, has been modeled redefining the donor and acceptor concentrations according to the following expressions:

$$
N_{\mathrm{D}}^{+}=\frac{N_{\mathrm{D}}}{1+2 \exp \left[0.045 /\left(K_{\mathrm{B}} T\right)\right]}
$$

for electrons; and

$$
N_{\mathrm{A}}^{-}=\frac{N_{\mathrm{A}}}{1+4 \exp \left[0.0438 /\left(K_{\mathrm{B}} T\right)\right]}
$$

for holes, along with the description of the equilibrium carrier concentration in terms of Fermi statistics.

\section{RESULTS}

The model described in the previous section has been applied to the investigation of electron and hole mobility as a function of impurity concentration and temperature.

The experiments show that the dependence of the electron mobility on temperature in intrinsic silicon differs from that of holes. In particular, at low temperatures $(T<100 \mathrm{~K})$ it is $\mu_{\mathrm{n}} \approx T^{-1.5}, \mu_{\mathrm{p}}$ $\approx T^{-2}$. On the other hand, at higher temperatures (up to $500 \mathrm{~K}$ ) several investigators agree on that the mobility of both species of carriers is well described by a relation of the form $\mu_{\mathrm{n}, \mathrm{p}}=\mu_{\max }(T /$ $300)^{-\gamma}$, with $2.2<\gamma<2.3$. The relative departure of the mobility laws in terms of the lattice temperature was initially ascribed to the different structure of the conduction and valence bands. However, after implementing the full-band structure in HARM, a quantitative agreement was found in a wide range of temperatures for the electron mobility only; on the contrary, the agreement of the hole mobility was acceptable at $300 \mathrm{~K}$, whereas a difference of about $20 \%$ was found at 200 and $400 \mathrm{~K}$. This behavior can be observed in Figures 1 and 2, where the intrinsic

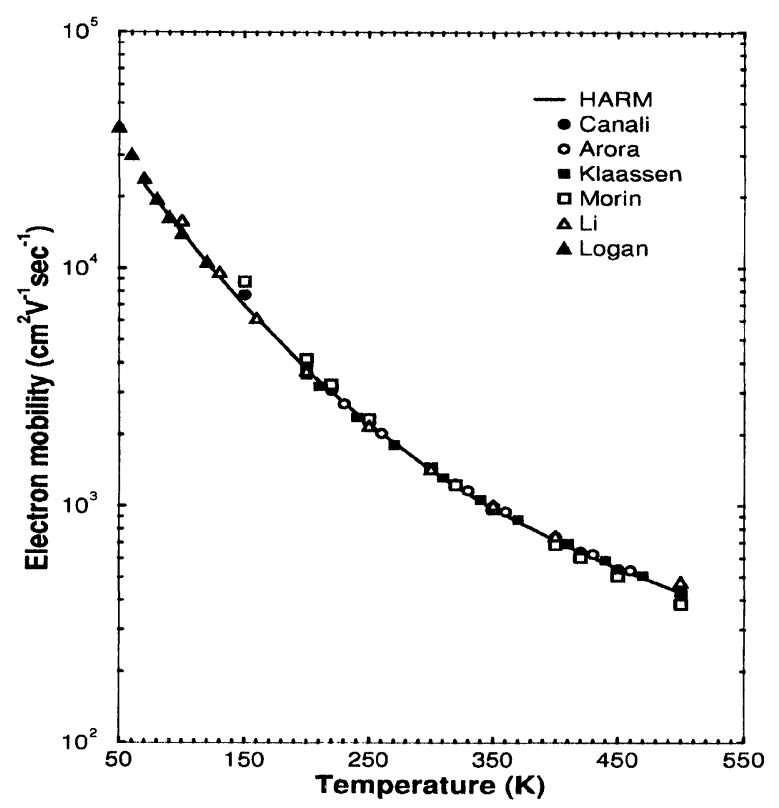

FIGURE 1 Experimental intrinsic electron mobility as a function of temperature compared with the results obtained with the HARM code. 


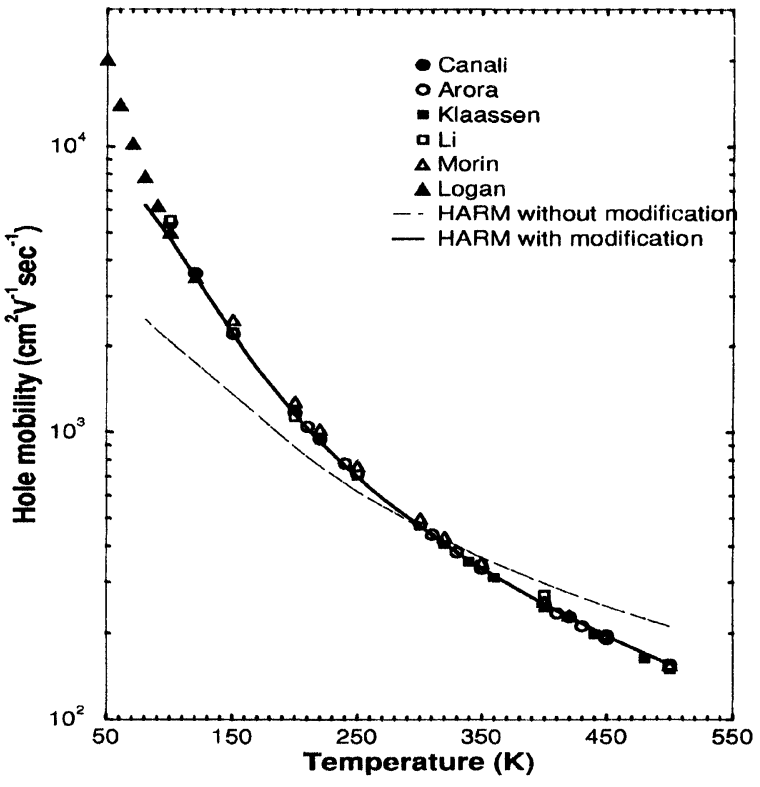

FIGURE 2 Experimental intrinsic hole mobility as a function of temperature compared with the results obtained with the HARM code. For the latter two different results are provided: the one labeled as "HARM (without modification)" refers to an acoustical-phonon scattering model with no dependence of the coupling constants on temperature; this dependence has been added in the results labeled as "HARM (with modification)".

mobility for electrons and holes as a function of temperature is reported. The curve for holes which corresponds to the physical model illustrated so far is labeled "HARM (without modification)".

Another effect whose temperature dependence proved relevant is the scattering with ionized impurities. To correctly describe such mechanism it is necessary to take the incomplete ionization into account, which becomes relevant when the total impurity concentration exceeds $10^{17} \mathrm{~cm}^{-3}$ and produces a significant lowering in the majority-carrier concentration with respect to that of the impurities. As already mentioned, incomplete ionization has been implemented into HARM, along with the description of the equilibrium carrier concentration in terms of Fermi statistics.

A final investigation is related to the phonon scattering, whose effect is modeled here following [4]. The values of the acoustical- and optical- phonon coupling constants were preliminarily fitted on the experimental data at $300 \mathrm{~K}$. Then, a model for the temperature dependence of the

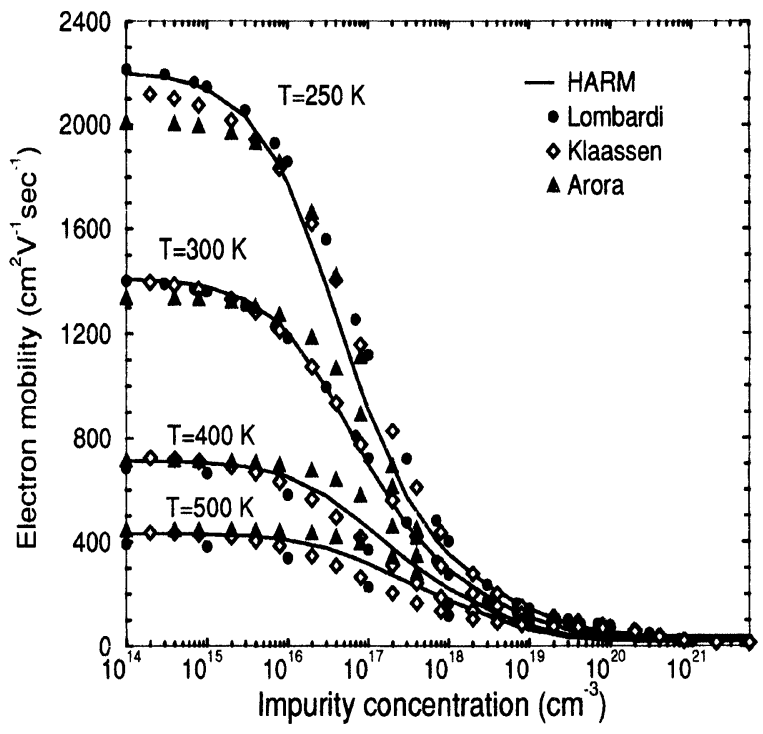

FIGURE 3 Experimental electron mobility as a function of impurity concentration at different temperatures compared with the results obtained with the HARM code.

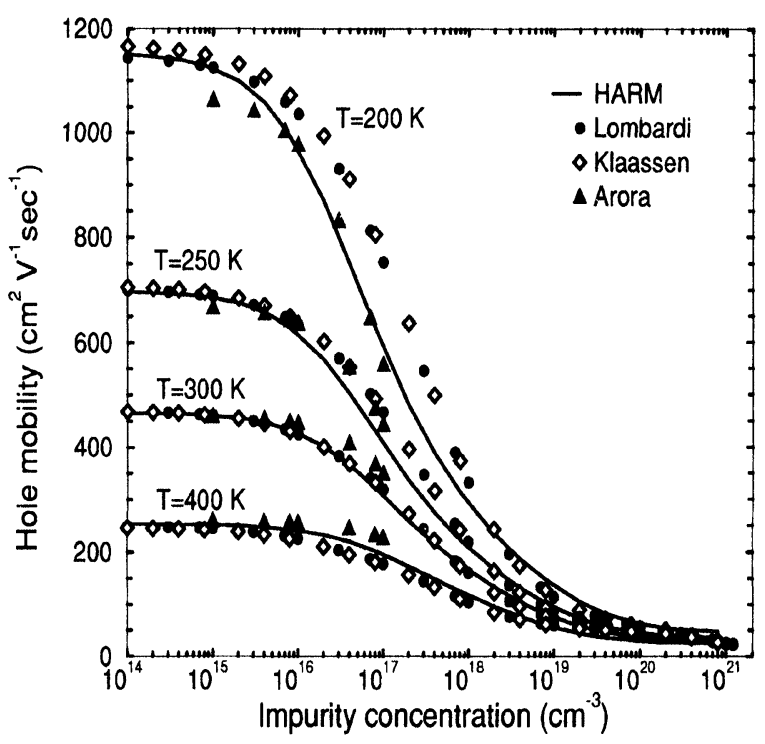

FIGURE 4 Experimental hole mobility as a function of impurity concentration at different temperatures compared with the results obtained with the HARM code. 
acoustical-phonon coupling constant has been implemented into HARM and the values of its parameters have been derived from the experiments. The result obtained with this additional dependence on temperature of the acousticalphonon coupling constant is indicated in Figure 2 with the label "HARM (with modification)".

A number of runs have been carried out to test the model described above in a typical range of temperatures as a function of impurity concentration. The comparison with experimentally-fitted curves of electron and hole mobility reported by different investigators is shown in Figures 3 and 4: in both cases a satisfactory agreement with experimental data has been obtained.

\section{References}

[1] Gnudi, A., Ventura, D., Baccarani, G. and Odeh, F. (1993). Two-dimensional MOSFET Simulation by Means of a Multidimensional Spherical Harmonics Expansion of the Boltzmann Transport Equation. Solid-State Electronics, 36(4), 575-581.

[2] Vecchi, M. C., Ventura, D., Gnudi, A. and Baccarani, G. (1994). Incorporating Full Band-Structure Effects in the Spherical-Harmonics Expansion of the Boltzmann Transport Equation. In H. S. Bennet and M. E. Law, editors, Proc. of the NUPAD V Conference, pp. 55-58, Honolulu, IEEE.

[3] Gnudi, A., Ventura, D. and Baccarani, G. Modeling Impact Ionization in a BJT by Means of a Spherical Harmonics Expansion of the Boltzmann Transport Equation. IEEE Trans. on CAD of ICAS, CAD-12(11), 1706-1713, November 1993.

[4] Jacoboni, C. and Reggiani, L. (1983). The Monte Carlo method for the solution of charge transport in semiconductors with applications to covalent materials. Reviews of Modern Physics, 55, 645.

[5] Haug, H. and Koch, S. W. (1993). Quantum Theory of the Optical and Electronic Properties of Semiconductors. World Scientific, Singapore.

[6] Moore, E. J. (1967). Quantum-transport theories and multiple scattering in doped semiconductors. I. Formal theory. Physical Review, 160(3), 607-617, August.

\section{Authors' Biographies}

Susanna Reggiani received the degree in Electrical Engineering from the University of Bologna, Italy, in 1997. Since April 1997 she has been working at the Department of Electronics (DEIS) of the same University in the field of the numerical simulation of semiconductor devices.

Maria Cristina Vecchi received the degree and the Ph.D. in Electrical Engineering from the University of Bologna, Italy, in 1990 and 1995, respectively. Since 1990 she has been working at the Department of Electronics (DEIS) of the same University in the field of the numerical simulation of semiconductor devices. In 1992-93 she visited the IBM T. J. Watson Research Center of Yorktown Heights, NY, working on higher-order transport models in semiconductors. In 1994 she visited the Integrated System Laboratories of the ETH, Zürich, working on the deterministic solution of the Boltzmann transport equation.

Massimo Rudan received a degree in Electrical Engineering in 1973 and a degree in Physics in 1976, both from the University of Bologna, Italy. He joined the Department of Electronics (DEIS) of the University of Bologna in 1975. From 1978 he has been teaching a course of Quantum Electronics in the Faculty of Engineering of the same University. Since 1983 he has been working in a group involved in numerical analysis of semiconductor devices. In 1986 he has been a visiting scientist at the IBM Thomas J. Watson Research Center at Yorktown Heights, NY, studying the discretization techniques for the higher-order moments of the Boltzmann Equation. In 1990, he was appointed Full Professor of Microelectronics at the University of Bologna. 

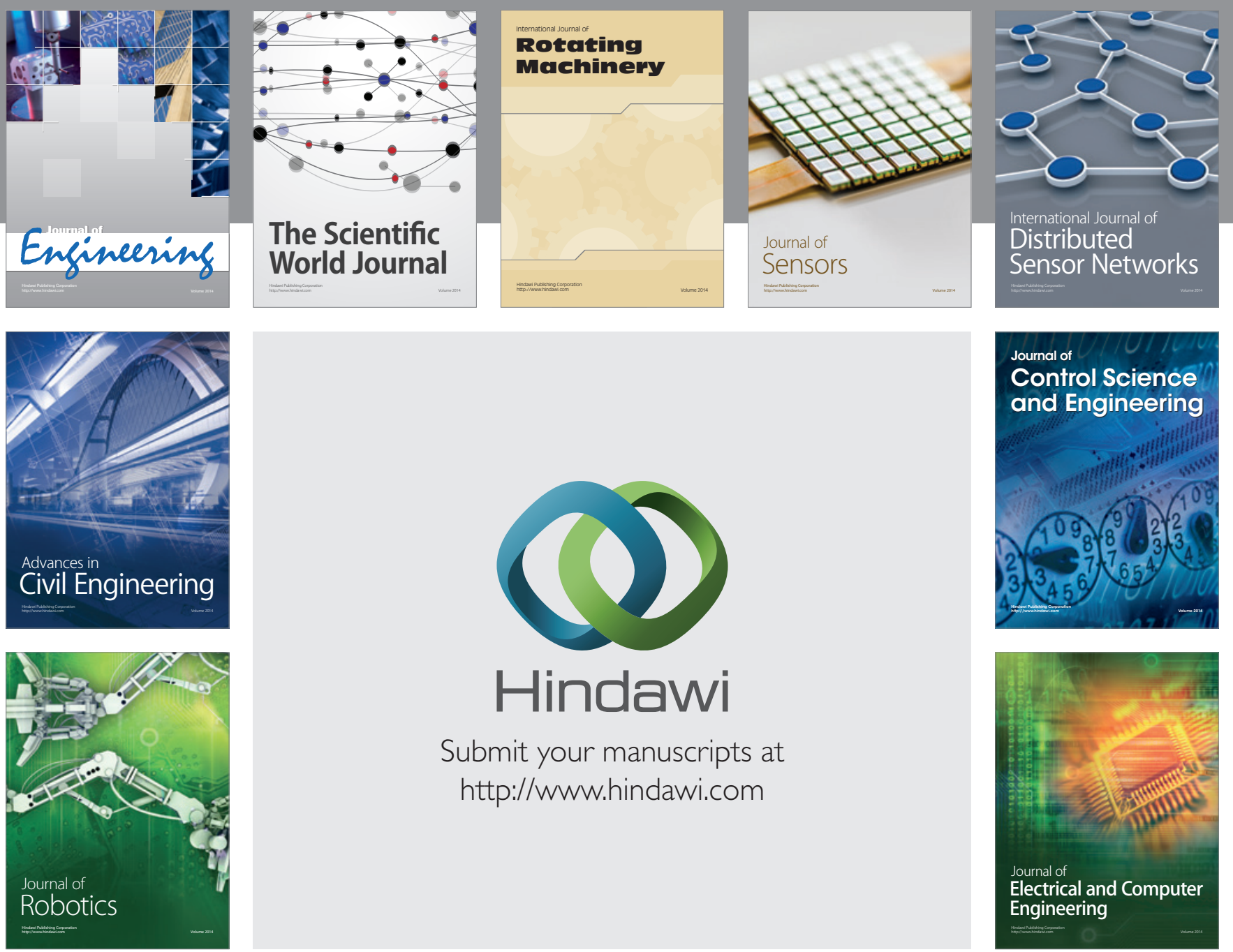

Submit your manuscripts at

http://www.hindawi.com
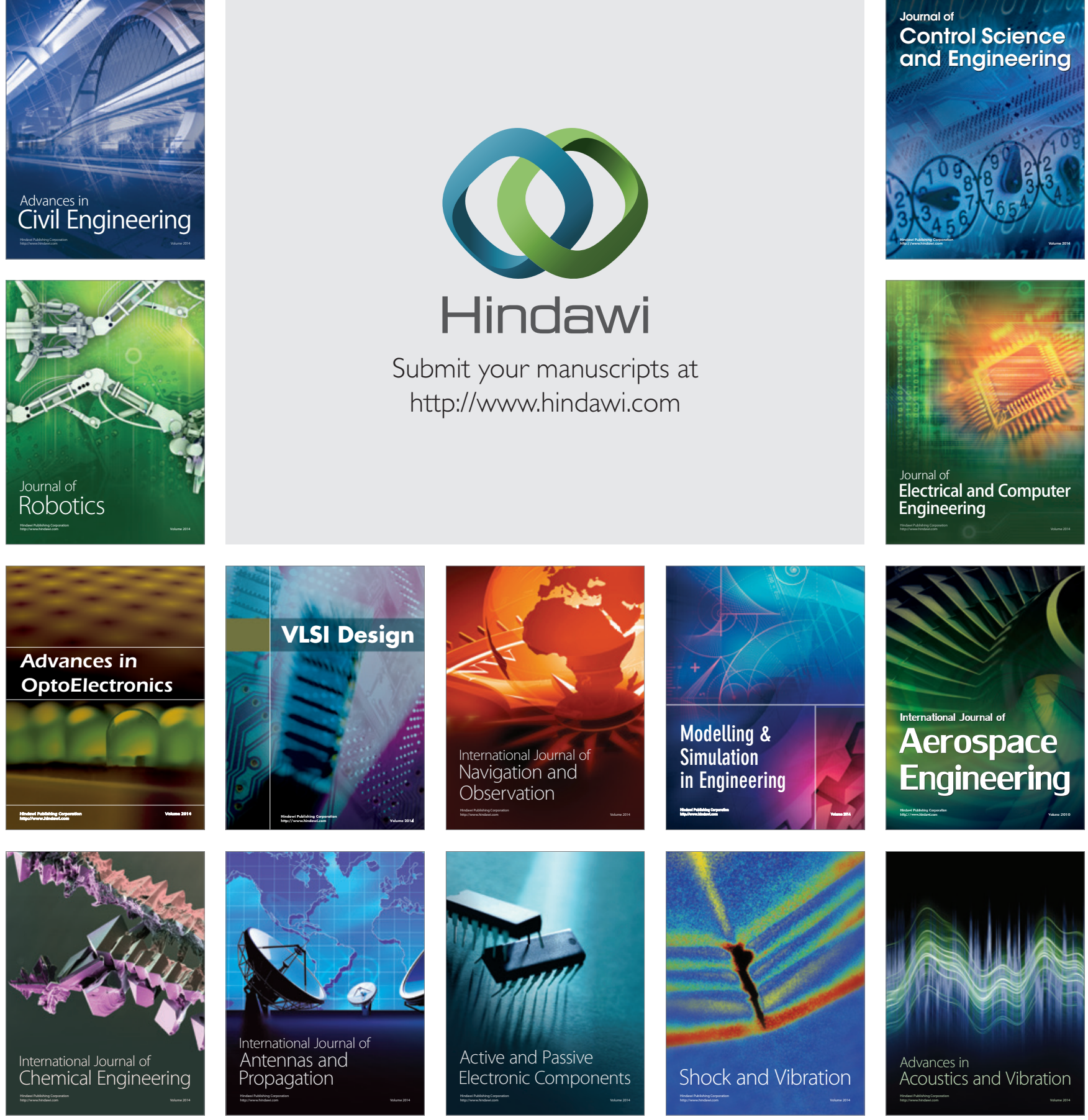